\title{
Animal model integration to AutDB, a genetic database for autism
}

\author{
Ajay Kumar, Rachna Wadhawan, Catherine Croft Swanwick, Ravi Kollu, Saumyendra N Basu, \\ Sharmila Banerjee-Basu*
}

\begin{abstract}
Background: In the post-genomic era, multi-faceted research on complex disorders such as autism has generated diverse types of molecular information related to its pathogenesis. The rapid accumulation of putative candidate genes/loci for Autism Spectrum Disorders (ASD) and ASD-related animal models poses a major challenge for systematic analysis of their content. We previously created the Autism Database (AutDB) to provide a publicly available web portal for ongoing collection, manual annotation, and visualization of genes linked to ASD. Here, we describe the design, development, and integration of a new module within AutDB for ongoing collection and comprehensive cataloguing of ASD-related animal models.

Description: As with the original AutDB, all data is extracted from published, peer-reviewed scientific literature. Animal models are annotated with a new standardized vocabulary of phenotypic terms developed by our researchers which is designed to reflect the diverse clinical manifestations of ASD. The new Animal Model module is seamlessly integrated to AutDB for dissemination of diverse information related to ASD. Animal model entries within the new module are linked to corresponding candidate genes in the original "Human Gene" module of the resource, thereby allowing for cross-modal navigation between gene models and human gene studies. Although the current release of the Animal Model module is restricted to mouse models, it was designed with an expandable framework which can easily incorporate additional species and non-genetic etiological models of autism in the future.
\end{abstract}

Conclusions: Importantly, this modular ASD database provides a platform from which data mining, bioinformatics, and/or computational biology strategies may be adopted to develop predictive disease models that may offer further insights into the molecular underpinnings of this disorder. It also serves as a general model for diseasedriven databases curating phenotypic characteristics of corresponding animal models.

\section{Background}

The dramatic rise in the prevalence of autism in recent years is of major public concern [1,2]. Autism (MIM 209850) is a broad-spectrum, multifactorial condition that onsets during the first years of life with a core triad of deficits in the areas of social communication, language development, repetitive activities and restricted range of interests (DSM IV, 1994). Due to the existence of a wide range of autism-related symptoms, this complex disorder is commonly described in the context of Autism Spectrum Disorders (ASD).

\footnotetext{
*Correspondence: sharmila@mindspec.org

MindSpec, 8280 Greensboro Dr, Suite 150, McLean, VA 22102, USA
}

A strong genetic component underlying ASD has been firmly established from various lines of studies [3-6] Genomic advances have led to the identification of hundreds of ASD candidate genes [7-11]. Recently, submicroscopic copy number variations (CNVs) were also strongly associated with ASD $[9,12,13]$. Furthermore, ASD is consistently associated with a number of specific genetic disorders caused by a single gene mutation, such as Fragile X Syndrome [14-17].

The high genetic heterogeneity of ASD poses an enormous challenge for understanding its etiology. For this reason, we have developed an autism gene database, AutDB, for ongoing curation of genes linked to the disorder [18]. AutDB is a disease-specific database model which curates information for all known ASD-linked
C Biomed Central

(c) 2011 Kumar et al; licensee BioMed Central Ltd. This is an Open Access article distributed under the terms of the Creative Commons Attribution License (http://creativecommons.org/licenses/by/2.0), which permits unrestricted use, distribution, and reproduction in any medium, provided the original work is properly cited. 
genes ranging from monogenic to risk-conferring candidates. Candidate genes are richly annotated for their relevance to autism and range of molecular functions. In this manner, AutDB serves as an up-to-date, annotated resource of ASD candidate genes which provides a bioinformatics framework for understanding the pathogenesis of ASD. It is widely used by the autism research community [19-22] and is also licensed to the Simons Foundation as SFARI Gene.

In recent years, various types of animal models based on ASD candidate genes/loci linked to autism in human studies have emerged, along with the creation of numerous etiologic animal models of autism. The rapid development of ASD animal models poses a major challenge for systematic analysis of their content. Herein, we describe the design, development, and integration of an animal models database module into AutDB which comprehensively curates and catalogs ASD-related animal models. In this new "Animal Model" module, we annotate animal models with a new standardized vocabulary of phenotypic terms developed by our laboratory in order to show the correspondence of heritable traits in animal models that are relevant for the diverse clinical manifestations of ASD. This resource contains a detailed phenotypic profile for each reported animal model, presented in a user-friendly format and keyword-searchable across all tables. Each model is manually curated, cited to its references in PubMed (http://www.ncbi.nlm.nih.gov/pubmed), and cross-referenced to its entries in three external databases: Entrez Gene (http://www.ncbi.nlm.nih.gov/gene), Mouse Genome Informatics (MGI; http://www.informatics.jax. org), and Allen Brain Atlas (http://www.brain-map.org/). Moreover, entries within the Animal Model module are linked to corresponding candidate genes in the original "Human Gene" module of AutDB, thereby allowing for cross-modal navigation between gene models and human gene studies. Although the current release of the Animal Model module is limited to mouse models, it was designed with an expandable framework which can easily incorporate additional species and non-genetic etiological models of autism in the future.

Herein, we describe the design, development, and integration of a new module within AutDB, a modular, disease-specific database previously developed by this research group [18]. This work provides a platform from which data mining, bioinformatics, and/or computational biology strategies may be adopted to develop predictive disease models that may offer further insights into the molecular underpinnings of ASD.

\section{Construction and Content}

\section{Data Extraction and Annotation}

Content of the Animal Model module originates entirely from published, peer-reviewed scientific literature and is manually annotated by expert biologists within our laboratory. In AutDB, an "animal model" is defined as an animal in which expression of an autism-associated gene has been manipulated. A comprehensive collection of mouse models was initially compiled from a search of the scientific literature using the PubMed database at NCBI http://www.ncbi.nlm.nih.gov/pubmed with the following keywords: gene symbol and aliases, mouse/mice, and knock-out/knock-in/transgenic. Additionally, mouse models listed in review articles on autism, along with cross-references therein, were mapped and incorporated into the PubMed search lists. Once a list of animal models has been generated, it is filtered using a set of specific criteria which maintains uniformity across the entire resource: First, only mouse model reports describing observations from behavioral or neurobiological tests are selected, even if the results are negative; Second, models showing a single phenotype (i.e., embryonic lethal) without further characterization are excluded; Third, the models need to be derived from a single gene that has linked to ASD in a human genetic study. Of note, future releases will support multi-gene constructs relevant for ASD. Finally, timed daily searches ensure that the Animal Model module maintains the most upto-date scientific content.

The challenge in developing an annotation strategy for ASD animal models is to include necessary and sufficient data fields that capture various attributes of the animal model, encompass various types of constructs (knock-outs, knock-ins, knock-downs, overexpression, conditional etc.), and include the wide spectrum of phenotypes reported about ASD in the scientific literature. Once a gene has been associated with ASD, multiple publications may report animal models using differing constructs of the same gene. To add to the complexity, many publications utilize a previously reported model to extend the characterization of the phenotype. Therefore, to navigate the intricacies of the animal models, we developed a classification system that allows us to faithfully represent the reported models. Every reported model is assigned a name that consists of the gene name, chronologically ordered model number, the model construct (allele type, such as Knock Out, Knock In, etc.), and finally the genotype (Homozygous, Heterozygous, Hemizygous). We also classify publications that report the first model of a gene as "primary" with every subsequent publication recorded as "additional". This allows us to differentiate the different models reported as well as highlight models that are being repeatedly utilized. A schematic representation of the distribution of the animal model data available in the literature is shown in Figure 1.

The Animal Model resource is constructed in a modular format with connections between sections that 


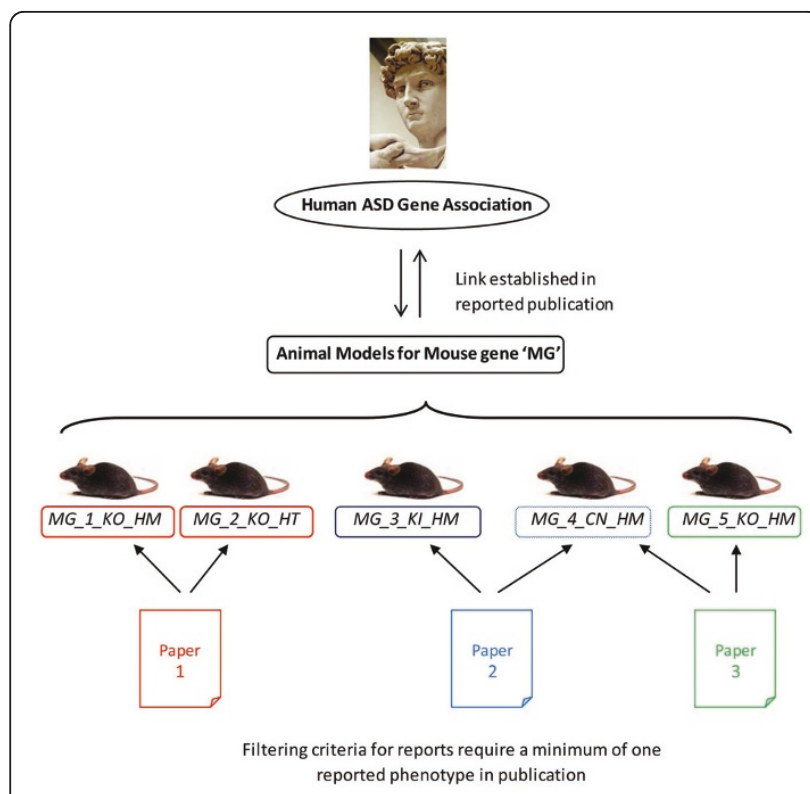

Figure 1 Data Extraction for the Animal Model Database. After a putative human candidate gene is associated to ASD and its mouse ortholog is used to create an animal model, we extract published data related to the animal model. This data consists of models curated from various reference articles. Moreover, each publication can report more than one model and/or multiple papers might report the same model, giving rise to complexity in classification.

maximize user-friendliness (Figure 2). This system allows users to obtain complete information about the model created for each autism-specific gene. Also embedded in the database is an integrated search engine that enables users to query across the annotations based not only on the gene, but also on the observed phenotypes and model types. It is specifically designed to be expandable to accommodate additional datasets or modules as they are added. The Animal Model dataset can be searched based on gene symbol, gene name, PhenoBase category, or model type. In addition to simple searches based on a single data field, advanced queries can be built by combinations of relevant data set specific key items with restrictions on queries by way of constraints. Therefore, the combination of comprehensive data and greater data connectivity and integration provides a powerful and useful disease-based resource for biologists.

\section{PhenoBase}

Importantly, our design of the new Animal Model module needed to address how the phenotypic profile observed in animal studies relates to the broad range of clinical manifestations of ASD reported in humans. The core behavioral domains of autism involving higher order human brain functions, such as social interaction and communications, can only be approximated in animal models. However, quantifiable and heritable traits in the mouse models can serve as markers providing mechanistic insight into the pathophysiology of the disease. Therefore, we developed an annotation model that attempts to capture and organize phenotypic data in clinically relevant domains in addition to the core behavioral features used in defining autism in humans. To this end, we developed "PhenoBase," a reference table which annotates models with new standardized phenotypic terms relevant to autism biology developed by our research team and reviewed by scientific experts on the Simons Foundation Advisory Board [23].

PhenoBase is a key component of the Animal Model module, serving as a repository of standardized phenotype terms and their definitions for annotating the animal models. To initiate the vocabulary, we first developed a high-level classification scheme encompassing 16 broad categories relevant to clinical presentations of ASD in humans (Table 1). These categories were derived from core behavioral features of ASD (social interactions \& communications, repetitive behavior), together with auxiliary features of autism (seizure, mental retardation, motor phenotype, sleep pattern) observed in humans. Additionally, phenotypic characteristics reported in animal models of candidate genes (i.e., maternal behavior, an abnormality in or lack of grooming, nursing, or retrieval of pups.) were also included. Lastly, information reported on the structure and function of these genes in the brain, in animal models, was grouped under three categories of neuroanatomy/ ultrastructure/cytoarchitecture, synaptic function, and molecular profile.

To expand the scope of the Animal Model resource, each term contained within the PhenoBase was expanded to encompass both Experimental Paradigm data as well as age of testing. For each model, the appropriate terms were classified as having changed (increased, decreased, or abnormal) or not changed, depending on reports from the corresponding reference article. To maintain consistency across models, unused categories were annotated as not reported. This annotation model not only provides a complete overview of the phenotype along with pertinent supplementary information, but also allows for comparison of various types of animal models developed for each candidate gene. Additionally, this framework allows the animal models can be evaluated and scored based on the number of the ASD-related phenotype observed in the model.

To prevent overlap within PhenoBase, each category consists of multiple terms with distinct definitions based on the observations/results of a particular test. This system of classification removes focus from the individual 


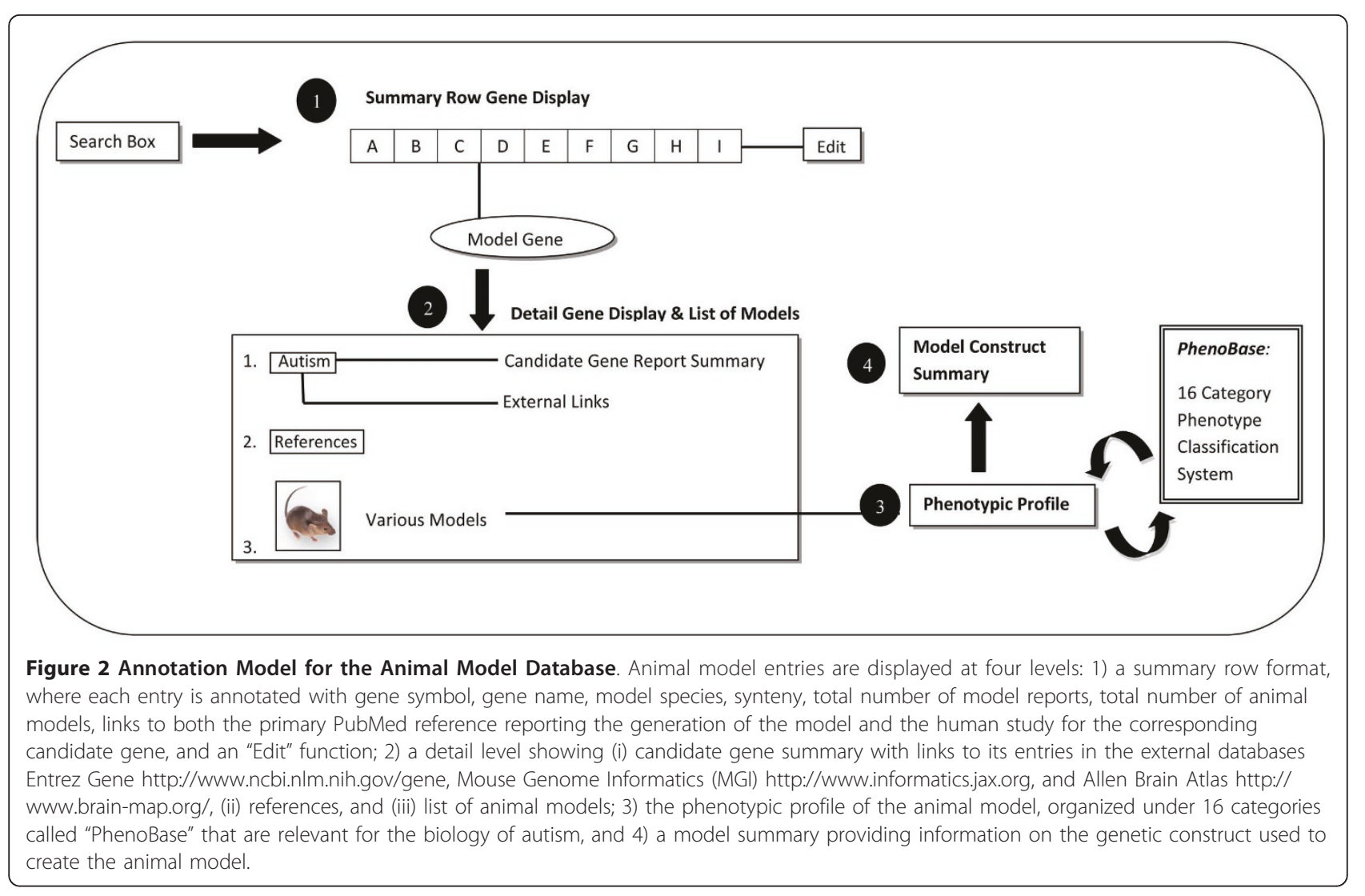

Table 1 PhenoBase.

\begin{tabular}{|c|c|c|c|}
\hline $\begin{array}{l}\text { Category } \\
\text { ID }\end{array}$ & PhenoBase Category & Relationship to Autism & Relevance to Autism \\
\hline 1 & General observations & & \\
\hline 2 & Social behavior & Core behavioral phenotype: Social interactions & DSM IV diagnostic criteria \\
\hline 3 & Communications & Core behavioral phenotype: Communications & DSM IV diagnostic criteria \\
\hline 4 & Repetitive behavior & $\begin{array}{l}\text { Core behavioral phenotype: Restricted interests \& } \\
\text { Repetitive behavior }\end{array}$ & DSM IV diagnostic criteria \\
\hline 5 & Maternal behavior & Broader phenotype of social memory, affect \& attachment & \\
\hline 6 & Motor phenotype & Auxiliary phenotype of ASD & Turner et al., 2001 [24] \\
\hline 7 & Sensory & Auxiliary phenotype of ASD & Geyer and Swedlow, 1998 [25] \\
\hline 8 & Learning \& memory & Auxiliary phenotype of ASD & \\
\hline 9 & Emotion & Hypothesis: Dysfunction of amygdala & Tsai, 1999 [26] \\
\hline 10 & Seizure & $25 \%$ cases & $\begin{array}{l}\text { Ballaban-Gil and Tuchman, } \\
2000 \text { [27] }\end{array}$ \\
\hline 11 & Circadian sleep/wake cycle & Auxiliary phenotype: sleep disturbances & $\begin{array}{l}\text { Harvey and Kennedy, } \\
2002[28]\end{array}$ \\
\hline 12 & Homeostasis & Unresolved & \\
\hline 13 & Inflammatory response & Auxiliary phenotype of ASD & \\
\hline 14 & Synaptic function & Hypothesis: Imbalance in E/I ratio & \\
\hline 15 & $\begin{array}{l}\text { Neuroanatomy/Ultra- structure/ } \\
\text { Cytoarchitecture }\end{array}$ & Macrocephaly & Piven et al., 1992 [29] \\
\hline 16 & Molecular profile & Unresolved & \\
\hline
\end{tabular}

Table depicting the 16 categories of PhenoBase, along with their relationships and relevance to autism. 
protocol, thereby limiting overlap across categories. To illustrate this system, we depict a representative sample of the terms contained within the "Learning/Memory/ Conditioning" and "Emotion" categories in Additional File 1. Although both categories contain terms with similar experimental paradigms (such as performance in different mazes), the reported results of the tests vary, warranting classification to two different categories.

Together, PhenoBase defines an integrated approach for autistic phenotypes, combining core behavioral domains of autism observed in humans with corresponding in-depth physiological and molecular characteristics reported in animal studies. The individual categories within PhenoBase are populated with terms extracted from autism-specific mouse model reports and review articles. An important consideration of our approach is to include robust terms to describe phenotypes for consistency in curation without losing their biological meaning or clinical relevance. The current version of PhenoBase includes $>100$ terms organized within the 16 major categories, and design of the table permits expansion of the categories with new terms and definitions as appropriate.

\section{Database Statistics}

Content of the Animal Model resource has significantly expanded over the past year (Figure 3). The database was first released in September 2009, at which time it contained 50 animal models. In January 2010, the database was updated to contain a total of 100 animal models. As of September 2010, the Animal Model module encompasses >200 models, with this number still

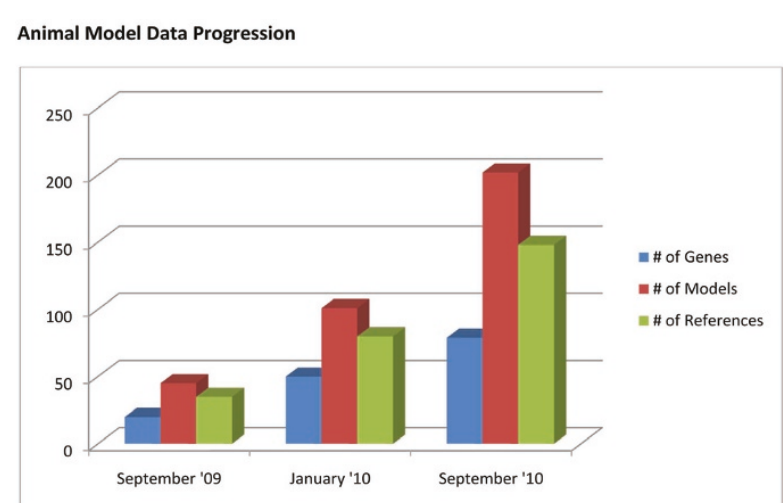

Figure 3 Expansion of the Animal Model Database. Content within the Animal Model database has rapidly accumulated over the past year. For example, the number of annotated animal models rose from 50 at the time of its beta release in September 2009 to 100 at the time of its official release in January 2010, and it now resides at $>200$ models. The number of corresponding genes and references within the Animal Model resource showed similar increases during this time period. growing. The number of annotated genes and references showed similar increases during this time period so that the Animal Model module is currently based on 79 genes and almost 150 references.

The current distribution of genetic model types within the Animal Models module is shown in Figure 4. As of September 1, 2010, the majority of genetic constructs used to create ASD-related animal models in this resource were Knock Out (63.4\%). By comparison, only a minority of animal models were created with Knock In $(6.9 \%)$, Conditional (12.4\%), or Other constructs (17.8\%).

\section{Database Implementation}

AutDB is a portal developed in JAVA on the J2EE platform on Linux with an RDBMS backend as its repository. AutDB is implemented in both Oracle and MySQL relational database management system where the biological information is stored. All data and images are stored in the relational database. The portal for AutDB is designed to be extensible where newer modules could be incorporated with relative ease by configuration. The application is deployed as a webapps in the Tomcat Application server connecting to the RDBMS. Connection pooling is provided by the Application Server, which greatly decreases the load on the system and enhances the performance. It also connects to the NLM database with the help of their DTDs and collects relevant information from the NLM databases for the end user. The editing, display and moderation interfaces (EDM) and automated load programs are used to display, query and input data into the AutDB system through an integrated web interface via the web browser. The EDM is an interactive, graphical interface used by scientists, curators and general users with varied role

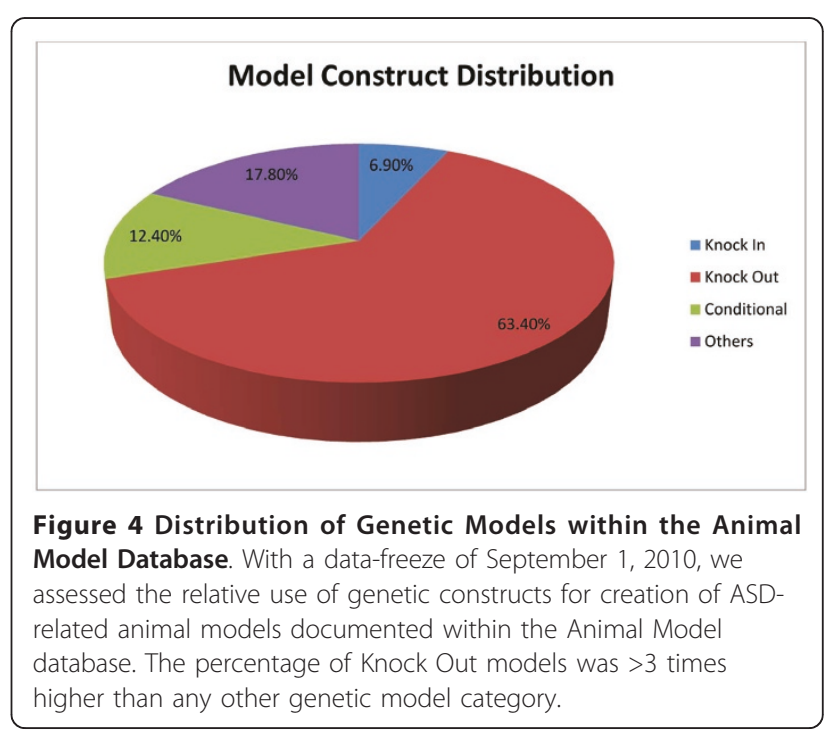


based privileges to perform their work. Thus, through EDM and automated loads, we acquire and integrate large amounts of data into a high quality, knowledgebase where the data is manually and automatically curated.

Public data access is also provided through the integrated web interface where users can interactively query and download slices of our data through a web browser.

\section{Utility}

The Animal Model module is seamlessly integrated within the gene portal so that the data can be searched and retrieved using a single search engine. This configuration essentially links two different types of datasets: Human Gene and Animal Model. From the search page, users can select the dataset and navigate based on their requirements. The information can be searched and displayed in several ways, including complex Boolean queries. Multiple search criteria allows for individualized searches by the end user. Searching by gene name or gene symbol retrieves a gene entry that can be displayed at four levels.

The first level of display is the summary row format. As developed for the Human Gene module of the resource, each animal model report pertaining to a candidate gene was extracted, counted for the number of studies and models, and collapsed under a single header representing the model gene entry. At the summary level, each entry row is annotated with gene symbol, gene name, model species, syntenic, total number of model reports, and total number of animal models, together with a primary PubMed reference reporting the generation of the model for the candidate gene. Additionally, within the summary line display, a link to the human ASD study for the corresponding gene is provided. Moreover, the summary line display includes an "Edit" functionality that allows registered AutDB users to enter new information about an animal model. Upon approval by our research team, this new data will be incorporated into the Animal Model module.

Each entry further displays at a detail level (Figure 5) showing: (i) ASD Candidate Gene, summarizing the gene and providing links to its entries in the external databases Entrez Gene http://www.ncbi.nlm.nih.gov/ gene, Mouse Genome Informatics (MGI) http://www. informatics.jax.org and the Allen Brain Atlas http:// www.brain-map.org/, (ii) References, providing links to its citations in PubMed http://www.ncbi.nlm.nih.gov/ pubmed, and (iii) Various Models, reporting the list of animal models related to that gene.

At the third level, in-depth phenotypic characterization of the model is provided using ASD-specific annotation specifically developed for this module. The data is represented in a tabular format divided into the 16 phenotypic categories which we coined "PhenoBase" (Table 1), to be described in the next section. Each animal model with a reported phenotype is hyperlinked to its corresponding entry in PhenoBase, where any variation in phenotype of the model is color coded differently from cases of no change, thus allowing maximum comprehension for users. Alongside PhenoBase, each animal model entry is annotated with information about the experimental paradigm used and age at testing so as to allow researchers to have maximum information to conduct comparative analysis or to replicate models. Finally, the fourth level of display provides the construct definition which defines the strain of origin of mice along with the methods used to create the model.

\section{Discussion}

Our annotation strategy involved the development of standardized terms and definitions specific to the biology of autism. The advantage of using such controlled vocabulary for defining phenotypes has been long recognized. However, a comprehensive collection of terms that is relevant for a complex human disorder with broad clinical manifestations, such as ASD, is a major challenge. With PhenoBase, we integrate core behavioral domains of autism observed in humans with corresponding in-depth physiological and molecular characteristics reported in animal studies. AutDB coupled with PhenoBase affords structured classification of a heterogeneous profile such as the phenotype data of mice. For example, multiple gene mutations that result in similar neuroanatomical changes can be filtered, thereby paving the way for elucidation of common/divergent pathways. This sort of comparison allows for consolidation of different sources of data in order to simplify data mining and analysis. By comparing results from the 16 categories of PhenoBase, researchers can more easily evaluate, score, and prioritize autism-related animal models for future ASD research.

PhenoBase is envisioned to be an ASD research community-based tool that is edited and updated by experts who are actually performing research on these mouse models. This initial draft of PhenoBase is anticipated to enable robust phenotyping of animal models and to provide a platform for further refining of the terms with precise definitions and additional attributes. For example, in the future, the models will be annotated with a series of attribute vocabulary terms that describe the quality, quantity, and character of each phenotypic term. Such refinement of PhenoBase will be accelerated by our incorporation of the "Edit" function in the summary line display of this module which allows outside researchers to provide new information about ASD animal models. In this manner, we are encouraging widespread participation from the ASD research community 


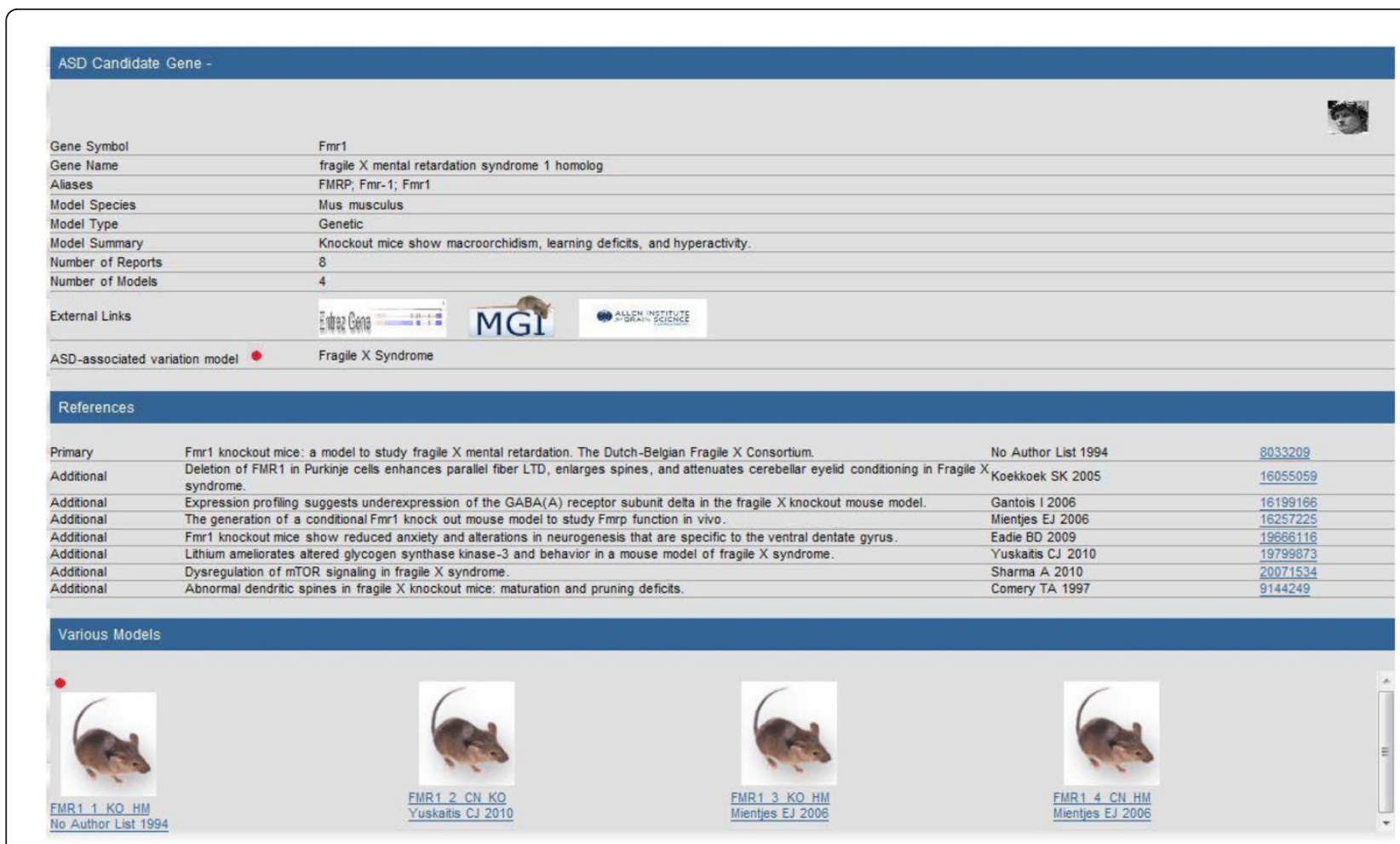

Figure 5 Detail Level Display of the Animal Model Database. At the second level of display, the new Animal Model resource provides a gene detail entry page showing (i) ASD Candidate Gene, summarizing the gene and providing links to its entries in the external databases Entrez Gene http://www.ncbi.nlm.nih.gov/gene, Mouse Genome Informatics (MGl) http://www.informatics.jax.org and the Allen Brain Atlas http://www. brain-map.org/, (ii) References, providing links to its citations in PubMed http://www.ncbi.nlm.nih.gov/pubmed, and (iii) Various Models, reporting the list of animal models related to that gene, where each animal model is assigned a model ID consisting of the Target Gene Symbol, chronological order number, construct type (Knock Out, Knock In, Conditional, Transgenic, Knock Down, etc.), and the genotype of the model (Homozygous, Heterozygous, Hemizygous). If the same model is reported in multiple publications, the model receives one identification number but each reference is listed and the phenotypic profile is pooled.

that will enhance AutDB as a tool for collective knowledge discovery.

The open nature of the Animal Model database implies easier expansion. In addition to adding increasing numbers of genetic models for ASD, its framework will allow us to incorporate non-genetic, etiologic models of ASD. It will also facilitate the incorporation of additional species of animal models. Moreover, the breadth of PhenoBase to encompass various terms does not limit it to a specific disease, but allows for implementation to many different disorders. For instance, Schizophrenia Gene (SZGene; http://www.szgene.org) currently includes an animal models resource but does not provide search capability similar to PhenoBase. Our development of PhenoBase permits phenotypes within the heterogeneous world of neurodevelopmental disorders to be directly compared using standardized vocabulary. With the advent of new technologies and increased identification of candidate genes for different disorders, disease-specific databases will prove to play a key role in disease biology.
With this new module of AutDB, we have created a framework that captures the phenotype of ASD animal models. This resource not only acts as a repository for disease-specific models but also allows for comparison across models. The resource has implications for the development of standards for data deposition, exchange. More importantly, it accelerates ASD research by promoting comparative analysis and prioritization of ASD animal models.

\section{Conclusions}

Overall, we describe the design, development, and integration of a new module within AutDB for ongoing collection and comprehensive cataloguing of animal models linked to ASD. The current version of this Animal Model module curates mouse models based on manipulation of a single gene linked to ASD. Data is displayed at summary and detail levels. Moreover, the detail level of display contains a novel repository of standardized phenotype categories and terms for annotating animal models called "PhenoBase." With the integration of the 
new Animal Model module, AutDB provides a platform from which data mining, bioinformatics, and/or computational biology strategies may be adopted to develop predictive disease models for ASD. It also serves as a general model for disease-driven databases to systematically curate animal model phenotypes.

\section{Availability and Requirements}

AutDB URL: http://www.mindspec.org/autdb.html

SFARI Gene URL: http://gene.sfari.org/

\section{Additional material}

Additional file 1: PhenoBase Categories Avoid Overlap by Distinct

Definitions of Terms. To prevent overlap within PhenoBase, each

category consists of multiple terms with distinct definitions based on the observations/results of a particular test. To illustrate this system, we

depict here a representative sample of the terms contained within the "Learning/Memory/Conditioning" and "Emotion" categories.

\section{Acknowledgements}

AutDB is funded by the Simons Foundation, which licenses it as SFARI Gene http://gene.sfari.org/. We also thank Dr. Dario Dieguez for his thoughtful edits of this manuscript.

\section{Authors' contributions}

AK performed most of the data curation and contributed to the database design. RW programmed the Java applications necessary for implementation of the database. CCS drafted the manuscript and critically analyzed the data. RK assisted in the design and implementation of the database. SNB led the design and implementation of the database. SBB guided and participated in all aspects of the project, including curation of initial database content, database design, and organizing the manuscript draft. All authors have read and approved the final manuscript

\section{Authors' Information}

SBB founded the nonprofit research organization MindSpec in 2006 and first released AutDB in 2007. She has over 20 years of expertise in neuroscience, bioinformatics, and systems biology.

\section{Competing interests}

The authors declare that they have no competing interests.

Received: 11 October 2010 Accepted: 27 January 2011

Published: 27 January 2011

\section{References}

1. Fombonne E: Epidemiology of autistic disorder and other pervasive developmental disorders. J Clin Psychiatry 2005, 66(Suppl 10):3-8.

2. Mulvihill B, Wingate M, Kirby RS, Pettygrove S, Cunniff C, Meaney FJ, Miller L, Robinson C, Quintana G, Kaiser MY, Lee LC, Landa R, Newschaffer C, Constantino J, Fitzgerald R, Daniels J, Giarelli E, PintoMartin J, Levy SE, Charles J, Nicholas J, Durkin M, Rice C, Baio J, Van Naarden Braun K, Yeargin-Allsopp M, Hepburn M, Garner N, Mancilla KC, Ratchford A, et al: Prevalence of autism spectrum disorders - Autism and Developmental Disabilities Monitoring Network, United States, 2006. MMWR Surveill Summ 2009, 58:1-20.

3. Bailey A, Le Couteur A, Gottesman I, Bolton P, Simonoff E, Yuzda E, Rutter M: Autism as a strongly genetic disorder: evidence from a British twin study. Psychol Med 1995, 25:63-77.

4. Le Couteur A, Bailey A, Goode S, Pickles A, Robertson S, Gottesman I, Rutter M: A broader phenotype of autism: the clinical spectrum in twins. J Child Psychol Psychiatry 1996, 37:785-801.
5. Chakrabarti S, Fombonne E: Pervasive developmental disorders in preschool children. JAMA 2001, 285:3093-3099.

6. Folstein SE, Rosen-Sheidley B: Genetics of autism: complex aetiology for a heterogeneous disorder. Nat Rev Genet 2001, 2:943-955.

7. Persico AM, Bourgeron T: Searching for ways out of the autism maze: genetic, epigenetic and environmental clues. Trends Neurosci 2006, 29:349-358.

8. Freitag CM: The genetics of autistic disorders and its clinical relevance: a review of the literature. Mol Psychiatry 2007, 12:2-22

9. Szatmari P, Paterson AD, Zwaigenbaum L, Roberts W, Brian J, Liu XQ, Vincent JB, Skaug JL, Thompson AP, Senman L, Feuk L, Qian C, Bryson SE, Jones MB, Marshall CR, Scherer SW, Vieland VJ, Bartlett C, Mangin LV, Goedken R, Segre A, Pericak-Vance MA, Cuccaro ML, Gilbert JR, Wright HH, Abramson RK, Betancur C, Bourgeron T, Gillberg C, Leboyer M, et al: Mapping autism risk loci using genetic linkage and chromosomal rearrangements. Nat Genet 2007, 39:319-328.

10. Yang MS, Gill M: A review of gene linkage, association and expression studies in autism and an assessment of convergent evidence. Int I Dev Neurosci 2007, 25:69-85.

11. Abrahams BS, Geschwind DH: Advances in autism genetics: on the threshold of a new neurobiology. Nat Rev Genet 2008, 9:341-355.

12. Sebat J, Lakshmi B, Malhotra D, Troge J, Lese-Martin C, Walsh T, Yamrom B, Yoon S, Krasnitz A, Kendall J, Leotta A, Pai D, Zhang R, Lee YH, Hicks J, Spence SJ, Lee AT, Puura K, Lehtimäki T, Ledbetter D, Gregersen PK, Bregman J, Sutcliffe JS, Jobanputra V, Chung W, Warburton D, King MC, Skuse D, Geschwind DH, Gilliam TC, et al: Strong association of de novo copy number mutations with autism. Science 2007, 316:445-449.

13. Marshall CR, Noor A, Vincent JB, Lionel AC, Feuk L, Skaug J, Shago M, Moessner R, Pinto D, Ren Y, Thiruvahindrapduram B, Fiebig A, Schreiber S, Friedman J, Ketelaars CE, Vos YJ, Ficicioglu C, Kirkpatrick S, Nicolson R, Sloman L, Summers A, Gibbons CA, Teebi A, Chitayat D, Weksberg R, Thompson A, Vardy C, Crosbie V, Luscombe S, Baatjes R, et al: Structural variation of chromosomes in autism spectrum disorder. Am J Hum Genet 2008, 82:477-488.

14. Rogers $S J$, Wehner $D E$, Hagerman R: The behavioral phenotype in fragile $\mathrm{X}$ : symptoms of autism in very young children with fragile $\mathrm{X}$ syndrome, idiopathic autism, and other developmental disorders. J Dev Behav Pediatr 2001, 22:409-417.

15. Jamain S, Quach H, Betancur C, Rastam M, Colineaux C, Gillberg IC, Soderstrom H, Giros B, Leboyer M, Gillberg C, Bourgeron T: Mutations of the X-linked genes encoding neuroligins NLGN3 and NLGN4 are associated with autism. Nat Genet 2003, 34:27-29.

16. Cohen D, Pichard N, Tordjman S, Baumann C, Burglen L, Excoffier E, Lazar G, Mazet P, Pinquier C, Verloes A, Héron D: Specific genetic disorders and autism: clinical contribution towards their identification. J Autism Dev Disord 2005, 35:103-116.

17. Durand CM, Betancur C, Boeckers TM, Bockmann J, Chaste P, Fauchereau F, Nygren G, Rastam M, Gillberg IC, Anckarsater H, Sponheim E, GoubranBotros H, Delorme R, Chabane N, Mouren-Simeoni MC, de Mas P, Bieth E, Rogé B, Héron D, Burglen L, Gillberg C, Leboyer M, Bourgeron T: Mutations in the gene encoding the synaptic scaffolding protein SHANK3 are associated with autism spectrum disorders. Nat Genet 2007, 39:25-27.

18. Basu SN, Kollu R, Banerjee-Basu S: : AutDB: a gene reference resource for autism research. Nucleic Acids Res 2009, 37:D832-836.

19. Crespi B, Stead P, Elliot M: Evolution in health and medicine Sackler colloquium: Comparative genomics of autism and schizophrenia. Proc Natl Acad Sci USA 2010, 107(Suppl 1):1736-1741.

20. Elia J, Gai X, Xie HM, Perin JC, Geiger E, Glessner JT, D'Arcy M, deBerardinis R, Frackelton E, Kim C, Lantieri F, Muganga BM, Wang L, Takeda T, Rappaport EF, Grant SF, Berrettini W, Devoto M, Shaikh TH, Hakonarson $\mathrm{H}$, White PS: Rare structural variants found in attentiondeficit hyperactivity disorder are preferentially associated with neurodevelopmental genes. Mol Psychiatry 2010, 15:637-646.

21. Gillis J, Mistry M, Pavlidis P: Gene function analysis in complex data sets using ErmineJ. Nat Protoc 2010, 5:1148-1159.

22. Toro R, Konyukh M, Delorme R, Leblond C, Chaste P, Fauchereau F, Coleman M, Leboyer M, Gillberg C, Bourgeron T: Key role for gene dosage and synaptic homeostasis in autism spectrum disorders. Trends Genet 2010, 26:363-372.

23. Banerjee-Basu S, Packer A: SFARI Gene: an evolving database for the autism research community. Dis Model Mech 2010, 3:133-135. 
24. Turner CA, Presti MF, Newman HA, Bugenhagen P, Crnic L, Lewis MH: Spontaneous stereotypy in an animal model of Down syndrome: Ts65Dn mice. Behav Genet 2001, 31(4):393-400.

25. Geyer MA, Swerdlow NR: Measurement of startle response, prepulse inhibition, and habituation. Curr Protoc Neurosci 2001, Chapter 8: Unit 8.7.

26. Tsail LY: Psychopharmacology in autism. Psychosom Med 1999, 61:651-665.

27. Ballaban-Gil K, Tuchman R: Epilepsy and epileptiform EEG: Association with autism and language disorder. Ment Retard Dev Disabil Res Rev 2000, 6(4):300-308.

28. Harvey MT, Kennedy CH: Polysomnographic phenotypes in developmental disabilities. Int I Dev Neurosci 2002, 20(3-5):443-448.

29. Piven J, Nehme E, Simon J, Barta P, Pearlson G, Folstein SE: Magnetic resonance imaging in autism: Measurement of the cerebellum, pons, and fourth ventricle. Biol Psychiatry 1992, 31(5):491-504.

\section{Pre-publication history}

The pre-publication history for this paper can be accessed here: http://www.biomedcentral.com/1755-8794/4/15/prepub

doi:10.1186/1755-8794-4-15

Cite this article as: Kumar et al.: Animal model integration to AutDB, a genetic database for autism. BMC Medical Genomics 2011 4:15.

\section{Submit your next manuscript to BioMed Central and take full advantage of:}

- Convenient online submission

- Thorough peer review

- No space constraints or color figure charges

- Immediate publication on acceptance

- Inclusion in PubMed, CAS, Scopus and Google Scholar

- Research which is freely available for redistribution

Submit your manuscript at www.biomedcentral.com/submit 